\title{
Detection and characterization of Pasteuria 16S rRNA gene sequences from nematodes and soils
}

\author{
Correspondence \\ A. V. Ogram \\ avo@mail.ifas.ufl.edu
}

\author{
Y. P. Duan, ${ }^{1}$ H. F. Castro, ${ }^{1}$ T. E. Hewlett, ${ }^{2}$ J. H. White ${ }^{2}$ and A. V. Ogram ${ }^{1,3}$ \\ ${ }^{1,3}$ Soil and Water Science Department ${ }^{1}$, and Microbiology and Cell Science Department ${ }^{3}$, \\ University of Florida, Gainesville, FL 32611-0290, USA \\ ${ }^{2}$ Entomos LLC, Gainesville, FL, USA
}

\begin{abstract}
Various bacterial species in the genus Pasteuria have great potential as biocontrol agents against plant-parasitic nematodes, although study of this important genus is hampered by the current inability to cultivate Pasteuria species outside their host. To aid in the study of this genus, an extensive 16S rRNA gene sequence phylogeny was constructed and this information was used to develop cultivation-independent methods for detection of Pasteuria in soils and nematodes. Thirty new clones of Pasteuria 16S rRNA genes were obtained directly from nematodes and soil samples. These were sequenced and used to construct an extensive phylogeny of this genus. These sequences were divided into two deeply branching clades within the low-G $+C, G$ ram-positive division; some sequences appear to represent novel species within the genus Pasteuria. In addition, a surprising degree of $16 \mathrm{~S}$ rRNA gene sequence diversity was observed within what had previously been designated a single strain of Pasteuria penetrans (P-20). PCR primers specific to Pasteuria $16 \mathrm{~S}$ rRNA for detection of Pasteuria in soils were also designed and evaluated. Detection limits for soil DNA were 100-10000 Pasteuria endospores (g soil) ${ }^{-1}$.
\end{abstract}

\section{INTRODUCTION}

Plant-parasitic nematodes cause significant yield losses of crops and other plants of economic value each year. Losses due to nematodes in 1994 were estimated to exceed $\$ 1.5$ billion for corn, soybean, wheat, cotton, peanut and vegetables combined (Koenning, 1999). There has been a steady decline in nematicides available for agronomic crops due to concern regarding environmental effects of these chemicals. As an example, methyl bromide is among the most effective means of controlling plant-parasitic nematodes. Methyl bromide is a potent degrader of ozone, however, and will be phased out of use in the United States by the year 2005 (Chitwood \& Deshusses, 2001). In response to declining options for control of nematodes, environmentally safe alternatives to control nematodes are of great interest. Among the most promising of these alternatives are biocontrol agents that specifically target these pests.

Pasteuria represents a genus of Gram-positive, endosporeforming bacteria whose species have been observed

Published online ahead of print on 12 July 2002 as DOI 10.1099/ ijs.0.02303-0.

The GenBank accession numbers for the sequences reported in this paper are AY081911-AY081940.

A table showing sequence similarities of all clones described in this paper is available as supplementary data in IJSEM Online (http:// ijs.sgmjournals.org). parasitizing the majority of economically important plantparasitic nematodes (Sayer \& Starr, 1988). Members of the genus Pasteuria have been reported from 323 nematode species belonging to 116 genera, including plant-parasitic, entomopathogenic, predatory and free-living nematodes (Chen \& Dickson, 1998). The potential of one species, Pasteuria penetrans, to be an effective biocontrol agent for Meloidogyne species (root-knot nematodes) has been demonstrated (Sayer \& Starr, 1988). In addition to P. penetrans, Pasteuria thornei and Pasteuria nishizawae have been reported to infect other plant-parasitic nematodes (Chen \& Dickson, 1998; Oostendorp et al., 1990). Variabilities in endospore size, endospore surface antigens and in the strength of endospore attachment to nematodes within the same population of Pasteuria species have been reported (Sharma \& Davies, 1996). Very limited information on the molecular biology, phylogeny and ecology of Pasteuria species is available due to the current difficulty in cultivating these bacteria outside of the host nematode (Chen \& Dickson, 1998). Development of a sensitive and specific tool for characterization of Pasteuria-nematode relationships, such as species-specific interactions between Pasteuria and nematodes, and for studying distribution of Pasteuria in soils would be of great benefit for evaluating the potential of this biocontrol agent as an alternative to nematicides (Stirling, 1991).

Analysis of $16 \mathrm{~S}$ rRNA gene sequences is a well-established 
Table 1. Clones of the Pasteuria 16S rRNA gene from nematodes and soils

Clones (ca. $1400 \mathrm{bp}$ ) amplified with universal primers are marked with an asterisk; all other clones (ca. $600 \mathrm{bp}$ ) were amplified with specific primers.

\begin{tabular}{|c|c|}
\hline Clone & Source \\
\hline $\mathrm{Nf}^{*}, \mathrm{Nf}^{*}, \mathrm{Nf} 9^{\star}, \mathrm{Nf} 21^{*}$ & $\begin{array}{l}\text { DNA extracted from endospores taken from female nematodes of } \\
\text { Meloidogyne arenaria Race } 1(\mathrm{P}-20) \text { collected from peanut plants } \\
\text { in North Central Florida and raised in tomato plants in a greenhouse }\end{array}$ \\
\hline SI1, SI6, SI10 & $\begin{array}{l}\text { Soil 1. Golf course at the Gainesville Golf and Country Club, } \\
\text { Gainesville, FL, USA, infested with sting nematodes (Belonolaimus longicaudatus) }\end{array}$ \\
\hline SII4, SII8, SII12 & $\begin{array}{l}\text { Soil 2. Nursery at the Gainesville Golf and Country Club, Gainesville, FL, USA, } \\
\text { infested with root-knot nematodes (Meloidogyne species) }\end{array}$ \\
\hline SV24, SV25, SV26, SV27, SV35, SV36 & $\begin{array}{l}\text { Soil 5. Golf course at the Gainesville Golf and Country Club, } \\
\text { Gainesville, FL, USA infested with lance nematode (Hoplolaimus species) }\end{array}$ \\
\hline
\end{tabular}

and sensitive tool for detection and phylogenetic analysis of bacteria (Stahl, 1997). Use of $16 \mathrm{~S}$ rRNA gene sequences to identify Pasteuria species and to assess the diversity of these bacteria within a nematode population or soil sample would be of great benefit in understanding nematode-parasite interactions and Pasteuria ecology. Development of $16 \mathrm{~S}$ rRNA-based phylogeny and detection systems requires compilation of $16 \mathrm{~S}$ rRNA gene sequences from a variety of representatives of the genus, yet only five 16S rRNA gene sequences for Pasteuria species could be retrieved from GenBank at the time of this study (Anderson et al., 1999; Atibalentja et al., 2000; Bekal et al., 2001; Ebert et al., 1996).

In this study, $16 \mathrm{~S}$ rRNA gene sequences were amplified from Pasteuria endospores extracted from Meloidogyne nematodes or soil samples and their phylogenetic relationships were established using maximum-parsimony analysis. Two sets of primers specific for the Pasteuria 16S rRNA gene were designed for detection and characterization of Pasteuria species from nematodes and nematode-infested soils, and a phylogeny of these sequences was developed. This work greatly extends previous $16 \mathrm{~S}$ rRNA phylogenies of Pasteuria, the most extensive of which include only two Pasteuria sequences (Anderson et al., 1999; Atibalentja et al., 1999).

\section{METHODS}

Bacteria sources and soils. P. penetrans P-20 endospores were collected from root-knot nematode Meloidogyne arenaria Race 1 as described by Anderson et al. (1999). In vitro cultures of Pasteuria originally collected from Pasteuria-infected $M$. arenaria Race 1 nematodes from peanut plants in North Central Florida were prepared as described by Gerber \& White (2001). Soils 1-5 (sources of clones) are described in Table 1. Soil 6 was taken from a residential garden in Gainesville, FL, USA. Endospore-filled Meloidogyne females were collected from greenhouse-cultivated tomato roots and ruptured with a plant tissue grinder to form an endospore suspension (Oostendorp et al., 1990).

DNA isolation. Pasteuria endospore DNA was extracted as described by Atibalentja et al. (2000). Soil and nematode DNA was isolated using an UltraClean soil DNA kit (MoBio) according to the manufacturer's instructions. For each sample, $0 \cdot 25 \mathrm{~g}$ soil was used for extraction. For quantitative detection of endospores, $P$. penetrans endospores were spiked into soil 6 at $10^{7}, 10^{6}, 10^{5}, 10^{4}, 10^{3}, 10^{2}, 10^{1}$ endospores $\mathrm{g}^{-1}$ and DNA was purified as described above.

PCR amplification and RFLP. 16S rRNA gene sequences were amplified with primers $27 \mathrm{~F}$ or 39F, and 1388R (Anderson et al., 1999; Atibalentja et al., 2000; Lane, 1991). Primers 617F, 654R and

Table 2. Primers for amplification of Pasteuria $16 \mathrm{~S}$ rRNA gene sequences

\begin{tabular}{|c|c|c|}
\hline Name & $5^{\prime} \rightarrow 3^{\prime}$ sequence & Reference \\
\hline $27 \mathrm{~F}$ & AGAGTTTGATCMTGGCTCAG & Lane (1991) \\
\hline $39 \mathrm{~F}$ & GCGGCGTGCCTAATACA & $\begin{array}{l}\text { Atibalentja et al. } \\
\text { (2000) }\end{array}$ \\
\hline $617 \mathrm{~F}$ & CGTGTRAGCGTTCGAAACGGT & This study \\
\hline 654R & TACCGCACTCAAGATGCACCG & This study \\
\hline $1166 \mathrm{R}$ & CGCCGGCTGTCTCTCCAA & This study \\
\hline $1388 \mathrm{R}$ & ACGGGCGGTGTGTACAAG & $\begin{array}{l}\text { Atibalentja et al. } \\
(2000)\end{array}$ \\
\hline
\end{tabular}




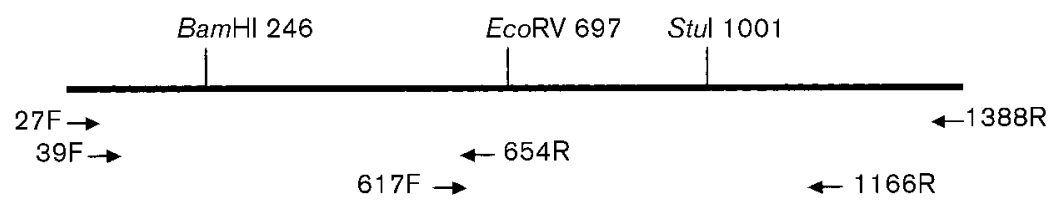

Fig. 1. Location of primers and restriction sites for characterization of Pasteuria 16S rRNA genes. Positions are indicated relative to the 16S rRNA gene sequence of the type strain of $P$. penetrans (GenBank accession no. AF077672).

1166R (Table 2) were designed for specific amplification of Pasteuria $16 \mathrm{~S}$ rRNA gene sequences on the basis of unique and consensus sequences among Pasteuria and related sequences. DNA was amplified using HotStarTaq Master Mix (Qiagen) in a GeneAmp PCR system 2400 (Perkin-Elmer Applied Biosystems) under the following conditions: initial enzyme activation and DNA denaturation of 15 min at $95^{\circ} \mathrm{C}$, followed by 35 cycles of $30 \mathrm{~s}$ at $94^{\circ} \mathrm{C}, 45 \mathrm{~s}$ at $61^{\circ} \mathrm{C}$ and $45 \mathrm{~s}$ extension at $72{ }^{\circ} \mathrm{C}$, and a final extension at $72^{\circ} \mathrm{C}$ for $7 \mathrm{~min}$. PCR products amplified with primers $27 \mathrm{~F}$ (or $39 \mathrm{~F}$ ) and $1388 \mathrm{R}$ were verified by digestion with $B a m \mathrm{HI}$ and EcoRV, products amplified with $39 \mathrm{~F}$ and $654 \mathrm{R}$ were verified by digestion with $\mathrm{BamHI}$, and products amplified with primers $617 \mathrm{~F}$ and $1166 \mathrm{R}$ were verified by digestion with StuI (Fig. 1). Amplification products were purified with the PCR Purification kit (QIAquick; Qiagen) and were cloned into the pCRII-TOPO cloning vector (Invitrogen). For RFLP assays, individual colonies were screened by direct PCR amplification of the target DNA fragment from Escherichia coli clones and the desired PCR products were subjected to RFLP analysis using the restriction enzyme HhaI and resolved by $2 \cdot 0 \%$ agarose gel electrophoresis.

Sequencing and phylogenetic analysis. 16S rRNA gene clones exhibiting different RFLP patterns were selected for sequencing. Selected amplification products were subjected to direct sequencing after cleaning with the QIAquick PCR Purification kit (Qiagen). Sequencing was carried out in the DNA Sequencing Core Laboratory at the University of Florida, Gainesville, Florida, USA. Six of the resulting sequences generated with universal primers were assembled to produce contigs of $\mathrm{ca} .1400$ bases and sequences of ca. 600 bases amplified with specific primers were initially aligned using the PILEUP function of the GCG (Genetics Computer Group, 1999). Final alignments were constructed by visual inspection using PAUP 4.0b8 (Swofford, 2001). Representative sequences of Pasteuria were aligned with other low-G $+\mathrm{C}$, Gram-positive bacterial species and maximum-parsimony analysis was conducted with Streptomyces griseus as the outgroup. The regions involving characters 1-30, 72-114 and 1463 to the end of the sequences were unalignable and therefore were not included in the maximum-parsimony analysis. Phylogenetic trees were built by a maximum-parsimony method using PAUP 4.0b8. Trees were constructed using heuristic searches with 10 random stepwise additions of taxa and by tree-bisection reconnection branch-swapping. The characters were weighted to give more weight to characters with lower levels of homoplasy. Bootstrap supports were obtained by heuristic searches of the reweighted trees with 100 or 1000 replicates.

Maximum-likelihood analyses were performed using the program TREE-PUZZLE version 5.0 obtained from http://www.tree-puzzle.de/ (Strimmer \& von Haeseler, 1996). Maximum-likelihood was performed using the Hasegawa-Kishino and Yano (Hasegawa et al., 1985) model of substitution with the transition/transversion ratio and nucleotide frequencies estimated from the dataset. The quartetpuzzling tree search algorithm with 10000 puzzling steps was used and the neighbour-joining method (Saitou \& Nei, 1987) was used for estimation of the tree parameters. A uniform-rate model of rate heterogeneity was assumed. Frequencies of puzzling steps in the quartet-puzzling tree search were used as a measure of confidence, which are highly correlated to bootstrap values (Strimmer \& von Haeseler, 1996). A sequence similarity matrix of all sequences described in this study was constructed using the distance matrix function in the RDP PHYLIP interface (Maidak et al., 2001).

\section{RESULTS AND DISCUSSION}

\section{PCR amplification and RFLP analysis of Pasteuria 16S rRNA genes}

Approximately 1400 bp of $16 \mathrm{~S}$ rRNA genes was amplified from DNA preparations of in vivo and in vitro (Gerber \& White, 2001) $P$. penetrans endospores resulting in clones Nf3, Nf7, Nf9, Nf21, NCf4, NCf8 (Table 1). These sequences shared $97 \cdot 5-100 \cdot 0 \%$ homology with previously reported $P$. penetrans $16 \mathrm{~S}$ rRNA gene sequences and were used to design PCR primers that would specifically amplify Pasteuria 16S rRNA genes. Based on consensus regions of these sequences, primer sets $617 \mathrm{~F} / 1166 \mathrm{R}$ and $39 \mathrm{~F} / 654 \mathrm{R}$ (Fig. 1) were thought to be Pasteuria-specific and were used in the subsequent amplification of Pasteuria sequences from soil and nematodes. A supplementary table showing sequence similarities of all clones described in this paper is available as supplementary material in IJSEM Online at http://ijs.sgmjournals.org/.

All Pasteuria sequences amplified with universal bacterial primers 27F (or 39F) and 1388R shared a few highly conserved restriction sites, including BamHI (nucleotide position 246 based on E. coli numbering), EcoRV (697) and StuI (1001) (Fig. 1). No related non-Pasteuria rRNA gene sequences included in the original alignment (Fig. 2) contained these digestion sites at these locations (as determined in silico; data not shown), suggesting that these restriction sites may be useful in preliminary identification of Pasteuria clones. Digestion of clones produced from amplification products using these primers provides a presumptive test for Pasteuria sequences before the DNA sequence is determined.

Amplification with primer set $39 \mathrm{~F} / 654 \mathrm{R}$ or $617 \mathrm{~F} / 1166 \mathrm{R}$ resulted in amplification of $16 \mathrm{~S}$ rRNA gene sequences from all soils and nematodes tested that were known to harbour Pasteuria (Fig. 3). All amplification products were initially verified by restriction digestion with either BamHI or StuI; 24 were sequenced (Table 1) and confirmed to be closely related to previously reported Pasteuria sequences (Anderson et al., 1999; Atibalentja et al., 2000).

The region amplified by primers $39 \mathrm{~F}$ and $654 \mathrm{R}$ is more variable between different clones than the region amplified by $617 \mathrm{~F}$ and $1166 \mathrm{R}$, such that PCR products amplified with 


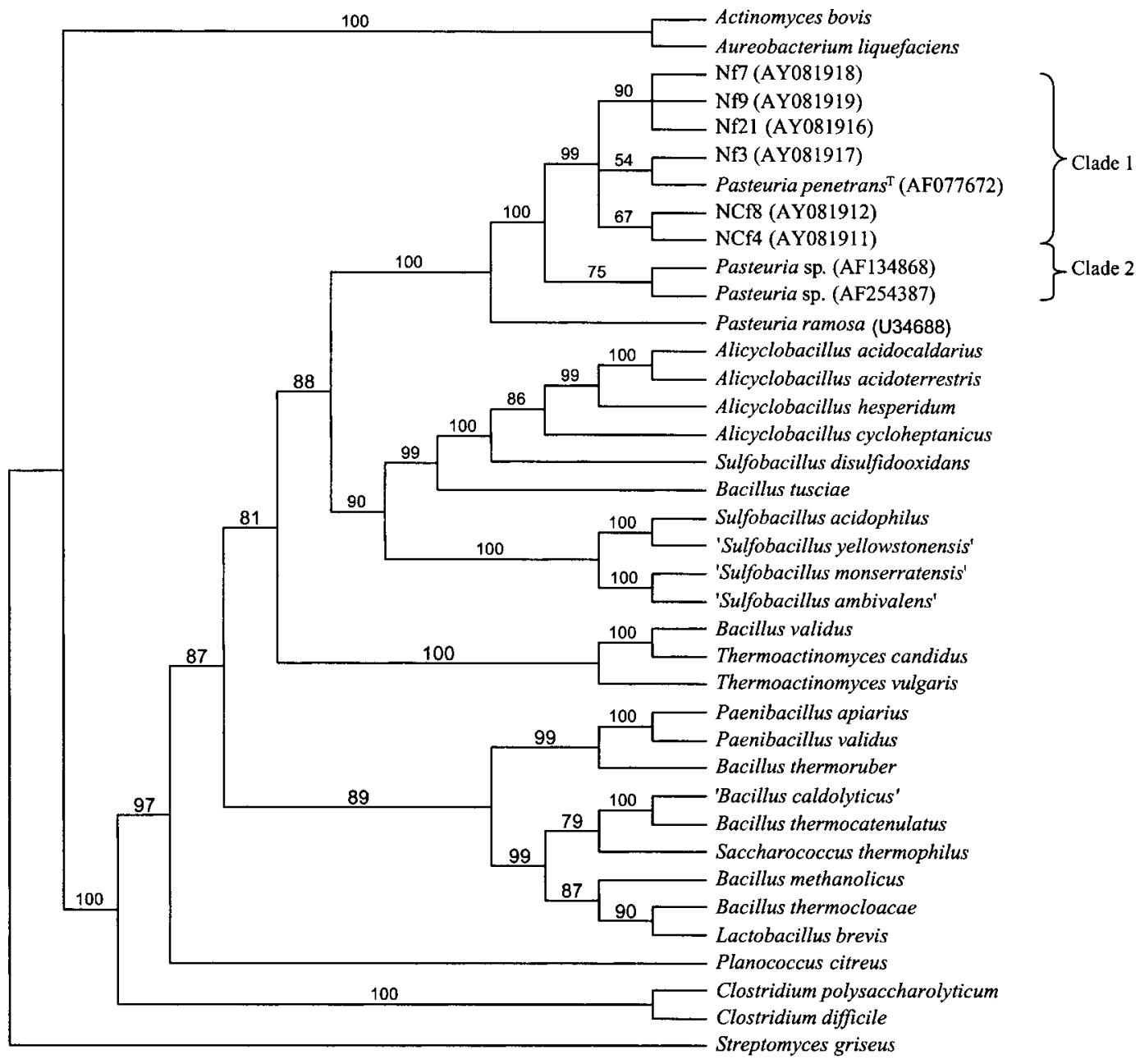

Fig. 2. Phylogenetic placement of Pasteuria species within the low- $\mathrm{G}+\mathrm{C}$, Gram-positive division. Analysis based on $1349 \mathrm{nt}$ sequences from positions 39-1388 (based on E. coli numbering) with Streptomyces griseus as the outgroup taxon. Numbers above nodes represent bootstrap values (100 replicates). GenBank accession numbers for sequences from 'Sulfobacillus yellowstonensis', 'Sulfobacillus montserratensis', 'Sulfobacillus ambivalens' and 'Bacillus caldolyticus' are AY007665, AY007663, AY007664 and Z26924, respectively.

these primers exhibited a greater diversity of RFLP patterns (Fig. 4). Although a variety of RFLP patterns was observed for clones from individual nematode and soil samples, the majority of clones from each sample exhibited a single dominant RFLP pattern. For example, only 5 out of 40 clones showed different RFLP patterns in the Np series (data not shown).

\section{Phylogenetic relationships between $P$. penetrans and other bacterial species}

To confirm the placement of Pasteuria within the low$\mathrm{G}+\mathrm{C}$, Gram-positive division and its association with the family Alicyclobacillaceae, a phylogenetic tree was constructed with maximum-parsimony from sequences available through GenBank and those from this study amplified from endospores and culture (Fig. 2). A total of 208 characters was excluded from the maximum-parsimony analysis yielding a matrix of 1389 characters. The matrix used in the phylogenetic analysis included 714 constant characters, 535 parsimony informative characters and 140 parsimony uninformative characters. The most parsimonious tree obtained had a tree length of 842 changes, a consistency index of $0 \cdot 65$, a retention index of $0 \cdot 81$, a rescaled consistency index of 0.53 and a homoplasy index of $0 \cdot 35$. According to maximum-parsimony, Pasteuria sequences were placed in a clade that contained previously described Pasteuria, Alicyclobacillus, Sulfobacillus and Bacillus species. This result is in general agreement with previously reported phylogenetic analyses (Anderson et al., 1999; Atibalentja et al., 2000; Ebert et al., 1996).

Pasteuria species have been characterized as belonging to the Alicyclobacillus group by GenBank and the 

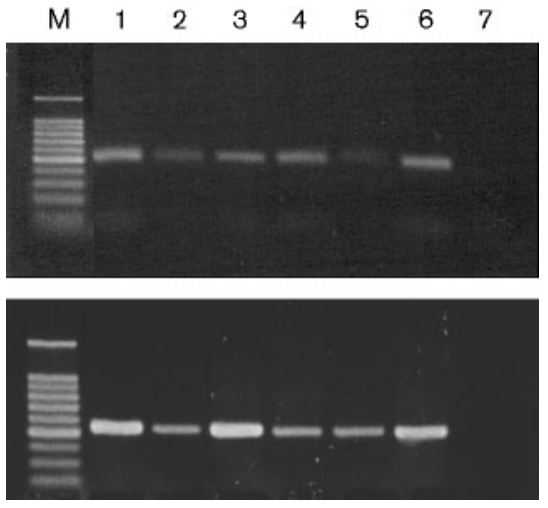

Fig. 3. PCR products amplified from nematode and soil samples using primers $39 \mathrm{~F}$ and $654 \mathrm{R}$ (top) and primers $617 \mathrm{~F}$ and 1166R (bottom). Lanes: $\mathrm{M}, 100 \mathrm{bp}$ molecular size marker (Promega); 1, DNA extracted from Meloidogyne arenaria Race 1 known to harbour $P$. penetrans; 2 , soil $1 ; 3$, soil $2 ; 4$, soil 3 ; 5 , soil $4 ; 6$, soil 5 ; and 7 , no template control.

Alicyclobacillaceae family by the taxonomic outline of the Archaea and Bacteria of Bergey's Manual of Systematic Bacteriology (http://www.cme.msu.edu/bergeys/). However, inclusion of additional Pasteuria, Alicyclobacillus and Sulfobacillus sequences resulted in a clear delimitation of a distinctive clade for Pasteuria from the rest of the Alicyclobacillus group (bootstrap value of 100). This distinction suggests that a re-evaluation of the placement of Pasteuria within the family Alicyclobacillaceae may be in order.

In our analysis, Pasteuria ramosa branched deeply within the Pasteuria clade and two additional major clades of Pasteuria could be distinguished (Fig. 2). One clade (P. penetrans clade 1; bootstrap support of $99 \%$ ) includes Pasteuria that parasitize root-knot nematodes, including the type species for P. penetrans (GenBank no. AF077672; Anderson et al., 1999 ) and our isolates that parasitize root-knot nematodes.

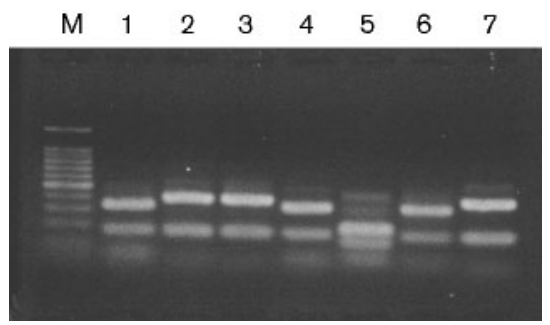

Fig. 4. Hhal digestion RFLPs of $39 F / 654 R$ amplification products from individual clones of the Pasteuria rRNA gene from nematodes or soils. Lanes: $\mathrm{M}, 100 \mathrm{bp}$ molecular size marker; 1, SV25; 2, SV24; 3, SV35; 4, Np3; 5, Np36; 6, Np4; and 7, Np16. SV series clones are from soil sample 5 (infested with lance nematodes); $\mathrm{N}$ series clones are from root-knot nematodes infected with $P$. penetrans.
The second clade (clade 2; bootstrap support of $75 \%$ ) includes Pasteuria that infect the cyst nematode Heterodera glycines (AF134868; Atibalentja et al., 2000; Sayre et al., 1991) and a strain that infects the sting nematode Belonolaimus longicaudatus (AF254387; Bekal et al., 2001).

Using primers $39 \mathrm{~F}$ and $654 \mathrm{R}$ designed for specific amplification of Pasteuria 16S rRNA gene sequences, 25 partial sequences of approximately 600 bp each of Pasteuria from different sources were included in a more comprehensive phylogenetic analysis of this bacterial group (Fig. 5). Thermoactinomyces dichotomicus was used as the outgroup. In this analysis, the alignment was edited to exclude the incomplete beginnings and ends of the sequences and yielded a matrix of 611 characters. The matrix used in phylogenetic analysis included 181 constant characters, 85 parsimony informative characters and 345 parsimony uninformative characters. The most parsimonious tree had a tree length of 476 changes, a consistency index of 0.94 , a retention index of $0 \cdot 84$, a rescaled consistency index of $0 \cdot 80$ and a homoplasy index of $0 \cdot 05$.

This analysis revealed diversity among the different Pasteuria sequences (Fig. 5). As in Fig. 2, P. ramosa was positioned on a deep branch of the phylogenetic tree and the two additional Pasteuria clades were separated. Bootstrap support for the $P$. penetrans clade (clade 1) was $99 \%$; however, support for clade 2 was quite low (67\%).

Clade 1 consists of sequences from strains (including the type strain of $P$. penetrans) known to infect root-knot nematodes and clones predominantly from soils infested with root-knot nematodes. It is not known at this time whether all clade 1 strains belong to the species $P$. penetrans. Barring $16 \mathrm{~S}$ rRNA gene sequence similarity of less than $97 \%$ (Stackebrandt \& Goebel, 1994), physiological and genetic studies are required to confirm that two strains are from different species. Due to the current lack of appropriate laboratory cultivation systems for Pasteuria, these tests are difficult at best. Possible differences in infectivity in nematodes are also difficult to assess at this time. These uncertainties will be greatly reduced when efficient systems for Pasteuria cultivation are developed (Gerber \& White, 2001).

Five subclades can be distinguished within clade 1. Subclades $1 \mathrm{a}, 1 \mathrm{~b}$ and $1 \mathrm{~d}$ have good bootstrap support values of 98, 94 and $93 \%$ respectively, suggesting that each is a distinctive clade within clade 1 . Subclades $1 \mathrm{c}$ and $1 \mathrm{e}$ are supported by lower bootstrap values, 78 and $80 \%$, respectively, which may compromise their phylogenetic position as individual clades within clade 1 . Ten sequences (Np and Nf series; Table 1) generated from endospores from a single race of root-knot nematode (Meloidogyne species) were originally thought to belong to a single strain previously designated $P$. penetrans $\mathrm{P}-20$ (Oostendorp et al., 1990; Anderson et al., 1999). In our analysis, ten P-20 sequences fell into three different subclades (1a, $1 \mathrm{~b}$ and $1 \mathrm{e}$ ). 

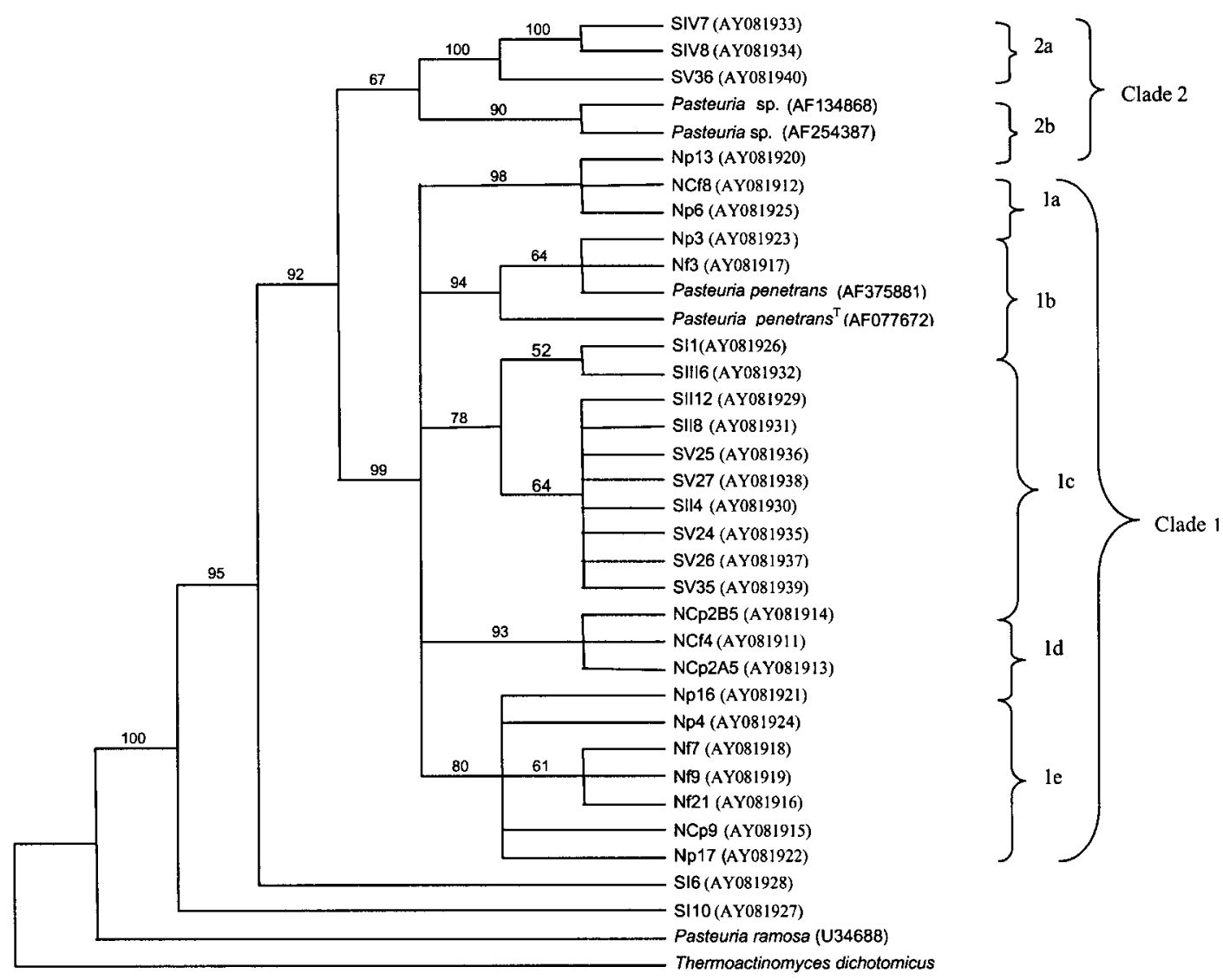

Fig. 5. Phylogenetic relationships among Pasteuria species. Phylogenetic reconstruction based on maximum-parsimony using heuristic search of 16S rRNA gene sequences (PAUP, version 4.0b8). Analysis based on ca. 600 nt sequences from positions 39-654 with Thermoactinomyces dichotomicus as the outgroup taxon. Numbers above nodes represent bootstrap values (1000 replicates).

Most sequences from soils 2 and 5 separated into a distinct subclade (1c).

Clade 2 includes sequences from clones taken from soils infested with a variety of nematodes, including root-knot, sting and cyst nematodes. This clade also includes previously described strains that infect cyst, lance and sting nematodes. It is not known at this time if clade 2 Pasteuria strains exclusively attack nematodes other than root-knot nematodes. A more complete understanding of relationships between phylogenetic groups of Pasteuria and the nematodes they infect would be very important in the accurate design and implementation of Pasteuria-based systems to control nematodes.

Clade 2 includes SIV7 and SIV8, clones obtained from soil 4 (which harboured sting nematodes), and SV36, obtained from soil 5 (known to harbour lance nematodes). Also included in clade 2 are sequences from a previously described cyst nematode-infecting Pasteuria (GenBank no. AF134868) (Atibalentja et al., 2000) and a sting nematode-infecting Pasteuria (AF254387) (Bekal et al.,
2001). Bootstrap support for this clade is low (67\%), such that confidence that clade 2 is a single clade is not high. It is possible that clade 2 should be divided into two individual clades and additional full sequences from this clade would help clarify the topology of the tree and define the clade. Clade 2 may be separated into at least two subclades (Fig. 5). Bootstrap support for separation of clade 2 into these two subclades is very strong and the sequence similarities between representatives of the two subclades are low (less than $93 \%$ ). As currently defined, $16 \mathrm{~S}$ rRNA gene sequence similarity may not be used to define species, but may be used to determine whether two strains do not belong to the same species if they share less than $97 \%$ similarity (Stackebrandt \& Goebel, 1994). On this basis, it is likely that subclades 2a and $2 \mathrm{~b}$ represent different species.

The number of characters used in this analysis was not high (85 parsimony informative characters) due to the relatively small amplification products and conserved regions within the amplicon. The robustness of branches formed in this analysis is therefore tenuous, although the topology of the tree did not change significantly when the near-full-length 
sequences presented in Fig. 2 were subjected to a similar analysis using the same region of sequence as those in Fig. 5 (analysis not shown). Robustness of the subclades discussed above will be tested as more sequences become available.

Maximum-likelihood analysis obtained by the quartetpuzzling method revealed a similar topology for the phylogenetic tree presented in Fig. 5, although some changes in the major clades were observed and supports for the main clades were lower (data not shown). Using this method, clade 1 has a support of $59 \%$, greater than the $50 \%$ recommended by the developers of the quartet-puzzling method. Clade 2 was not supported and subclades $2 \mathrm{a}$ and $2 \mathrm{~b}$ became individual clades. Inside clade 1 , each of the subclades exhibited frequencies of puzzling steps in the range 85-93, confirming the subclades achieved by maximumparsimony. Moreover, subclades $1 \mathrm{c}$ and le received supports of 89 and $93 \%$, respectively, possibly reaffirming their compromised identities as subclades in the maximumparsimony analysis.

\section{PCR detection of Pasteuria in soils and nematodes with Pasteuria-specific primers and their use in detection of Pasteuria in soils}

The Pasteuria-specific PCR primers developed as part of this study were also evaluated for specific detection of Pasteuria in soils and nematodes. Detection and enumeration of Pasteuria will facilitate studies to determine the efficacy of their commercial use as biocontrol agents as new systems for laboratory cultivation of Pasteuria are developed (Gerber \& White, 2001). Detection of sequences in serial dilutions of endospores added to soil 6 was consistently several orders of magnitude lower with primer set 617F/1166R than with 39F/ 654R (data not shown). The lowest detection limit for $P$. penetrans $16 \mathrm{~S}$ rRNA genes in soil 6 using primer set $617 \mathrm{~F}$ and $1166 \mathrm{R}$ was 100 endospores (g soil) ${ }^{-1}$ (Fig. 6). The primer sets proved to be specific for Pasteuria strains, as determined by sequence analysis (data not shown).

For initial screening (presumptive confirmation) of $16 \mathrm{~S}$ rRNA gene clones prior to sequencing, PCR products can be verified by digestion with the restriction enzymes BamHI or StuI, depending on the primer set used. All Pasteuria sequences reported to date, including those found in this study, have a single BamHI site in the $39 \mathrm{~F} / 654 \mathrm{R}$

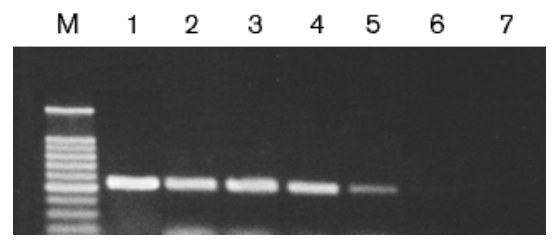

Fig. 6. PCR detection of $P$. penetrans spiked into soil 6 . Primer set $617 \mathrm{~F} / 1166 \mathrm{R}$ was used. Serial dilutions of endospores spiked into $1 \mathrm{~g}$ soil: $1,10^{7} ; 2,10^{6} ; 3,10^{5} ; 4,10^{4}$; $5,10^{3} ; 6,10^{2}$; and 7,$0 ; \mathrm{M}, 100$ bp molecular size marker; amplification product and a single StuI site in the 617F/ 1166R amplification product. For differentiation between different Pasteuria clones, digestion of individual 39F/654R products with $H$ haI is more informative than digestion of $617 \mathrm{~F} / 1166 \mathrm{R}$ due to greater sequence variability in this region between different clones. Evaluation of PCR primers for specific amplification and detection of these individual clades and subclades is currently being carried out.

In addition to providing information on the phylogeny of this potentially important biocontrol agent, the work presented here should form the basis for further studies that will elucidate the distribution and concentration of Pasteuria in soils and nematodes, and assist in defining host ranges of various strains and species of Pasteuria. Previous studies have shown that broader groups of Pasteuria exhibit preferences with regard to host nematodes (Davies et al., 1994). Additional studies are now needed to define host ranges of the various clades and subclades and to define potentially different species within these clades. Ultimately, however, a complete understanding of the relationship between Pasteuria and their nematode hosts will require an efficient system for laboratory cultivation.

\section{ACKNOWLEDGEMENTS}

This work was partially funded by a grant from the National Science Foundation.

\section{REFERENCES}

Anderson, J. M., Preston, J. F., Dickson, D. W., Hewlett, T. E., Williams, N. H. \& Maruniak, J. E. (1999). Phylogenetic analysis of Pasteuria penetrans by $16 \mathrm{~S}$ rRNA gene cloning and sequencing. J Nematol 31, 319-325.

Atibalentja, N. G., Noel, G. R. \& Domier, L. L. (2000). Phylogenetic position of the North American isolate of Pasteuria that parasitizes the soybean cyst nematode, Heterodera glycines, as inferred from the $16 \mathrm{~S}$ rDNA sequence analysis. Int J Syst Evol Microbiol 50, 605-613.

Bekal, S., Bomeman, J., Giblin-Davis, R. M. \& Becker, J. O. (2001). Phenotypic and molecular analysis of a Pasteuria strain parasitic to the sting nematode. J Nematol 33, 110-115.

Chen, Z. X. \& Dickson, D. W. (1998). Review of Pasteuria penetrans: biology, ecology, and biological control potential. J Nematol 30, 313-340.

Chitwood, D. E. \& Deshusses, M. A. (2001). Development of a methyl bromide collection system for fumigated farmland. Environ Sci Technol 35, 636-642.

Davies, K. G., Redden, M. \& Pearson, T. K. (1994). Endospore heterogeneity in Pasteuria penetrans related to adhesion to plantparasitic nematodes. Lett Appl Microbiol 19, 370-373.

Ebert, D., Rainey, P., Embley, T. M. \& Scholz, D. (1996). Development, life cycle, ultrastructure and phylogenetic position of Pasteuria ramosa Metchnikoff 1888: rediscovery of an obligate endoparasite of Daphnia magna Straus. Philos Trans $R$ Soc Lond B351, 1689-1701.

Genetics Computer Group (1999). Wisconsin Package version 10.0 (CGC). Madison, WI, USA. 
Gerber, J. F. \& White, J. H. (2001). Materials and methods for the efficient production of Pasteuria. Patent WO0111017.

Hasegawa, M., Kishino, H. \& Yano, K. (1985). Dating of the humanape splitting by a molecular clock of mitochondrial DNA. J Mol Evol 22, 160-174.

Koenning, S. R. (1999). Survey of crop losses in response to phytoparasitic nematodes in the United States for 1994. Nematology 31, 587-618.

Lane, D. J. (1991). 16S/23S rRNA sequencing. In Nucleic Acid Techniques in Bacterial Systematics, pp. 115-175. Edited by E. Stackebrandt \& M. Goodfellow. Chichester: Wiley.

Maidak, B. L., Cole, J. R., Lilburn, T. G. \& 7 other authors (2001). The RDP-II (Ribosomal Database Project). Nucleic Acids Res 29, 173-174.

Oostendorp, M., Dickson, D. W. \& Mitchell, D. J. (1990). Host range and ecology of isolates of Pasteuria spp. from the southern United States. J Nematol 23, 525-531.

Saitou, N. \& Nei, M. (1987). The neighbor-joining method: a new method for reconstructing phylogenetic trees. Mol Biol Evol 4, 406-425.

Sayer, R. M. \& Starr, M. P. (1988). Bacterial diseases and antagonisms of nematodes. In Diseases of Nematodes, pp. 59-101. Edited by G. O. Poinar, Jr \& H.-B. Jonson. Boca Raton, FL: CRC Press.
Sayre, R. M., Wergin, W. P., Schmidt, J. M. \& Starr, M. P. (1991). Pasteuria nishizawae sp. nov., a mycelial and endospore-forming bacterium parasitic on cyst nematodes of genera Heterodera and Globodera. Res Microbiol 142, 551-564.

Sharma, S. B. \& Davies, K. G. (1996). Characterization of Pasteuria isolated from Heterodera cajani using morphology, pathology and serology of endospores. Syst Appl Microbiol 19, 106-112.

Stackebrandt, E. \& Goebel, B. M. (1994). Taxonomic note: a place for DNA-DNA reassociation and 16S rRNA sequence analysis in the present species definition in bacteriology. Int J Syst Bacteriol 44, 846-849.

Stahl, D. A. (1997). Molecular approaches for the measurement of density, diversity and phylogeny. In Manual of Environmental Microbiology, pp. 102-114. Edited by C. J. Hurst, G. R. Knudsen, M. J. McInerney, L. D. Stetzenbach \& M. V. Walter. Washington, DC: American Society for Microbiology.

Stirling, G. R. (1991). Biological Control of Plant-parasitic Nematodes: Progress, Problems and Prospects. Wallingford: CAB International.

Strimmer, K. \& von Haeseler, A. (1996). Quartet puzzling: a quartet maximum likelihood method for reconstructing tree topologies. $\mathrm{Mol}$ Biol Evol 13, 964-969.

Swofford, D. L. (2001). PAUP: phylogenetic analysis using parsimony. Version 4.0b8. Sunderland, MA: Sinauer Associates. 